\title{
Morphological and Production Changes in Stressed Red Yeasts Monitored Using SEM and Raman Spectroscopy
}

\author{
K. Hrubanova ${ }^{1}$, O. Samek ${ }^{1}$, A. Haronikova ${ }^{2}$, S. Bernatova ${ }^{1}$, P. Zemanek ${ }^{1}$, I. Marova ${ }^{2}$, V. Krzyzanek ${ }^{1}$ \\ 1. Institute of Scientific instruments, The Czech Academy of Sciences, v.v.i., Brno, Czech Republic. \\ 2. Centre for Material Research, Brno University of Technology, Brno, Czech Republic.
}

We report on our investigations of the influence of different cultivation conditions on lipids, using scanning electron microscopy (SEM) and Raman spectroscopy techniques. Here, SEM uses electron beam to gain information about morphology of cells which reflects cells response on the applied stress. Consequently, Raman spectroscopy [1] was used for the determination of carotenoids and lipids present in the biomass. Thus, our study targets some factors which could lead to efficient industrial production of carotenoids and lipids in selected biotechnological production.

Applied stress and other environmental factors can induce changes in cell composition, metabolism and physiology [2]. In our investigations we focused on red/carotenogenic yeasts which also produce high amount of lipidic compounds. Carotenoids are membrane-bound lipid-soluble pigments, which can act as effective antioxidants and scavenge singlet oxygen. In red yeast controlled physiological and nutrition stress can be used for enhanced pigment and also oil production. Namely, in our experiments osmotic stress was induced in the two different media with different carbon to nitrogen $(\mathrm{C} / \mathrm{N})$ ratio which led to production of yeast lipids or carotenoids. Note, that also morphology of cells can be changed. Both media contain the same: salts, glucose and ammonium sulfate with different $\mathrm{C} / \mathrm{N}$ ratio. First growth medium (medium 1) indicate a positive results to carotenoids production and second medium (medium 2) with high $\mathrm{C} / \mathrm{N}$ ratio lead to an increased lipid production. Our experimental results are presented in Figure 1 and Figure 2.

For SEM imaging the samples of Cystofilobasidium infirmominiatum were prepared in the following way: (i) the samples were cultivated aerobically at $25^{\circ} \mathrm{C}$ in the two different media for 144 hours. (b) cells suspensions were fixed in $2.5 \%$ glutaraldehyde in PBS for 2 hours and 30 min in $1 \% \mathrm{OsO}_{4}$, dehydrated by ethanol series and dried in HDMS on the glass slides. Both images of prepared samples were scanned without any metal coating at electron beam energy $1 \mathrm{keV}$ and beam current $6.3 \mathrm{pA}$ in SEM Magellan (FEI). The samples of Sporobolomyces shibatanus were cultivated as mentioned above and observed by cryo scanning electron microscopy (cryo-SEM). The sample was quickly frozen in liquid nitrogen, moved into a vacuum chamber (ACE600, Leica Microsystems) where it was freezefractured and sublimated at $-95^{\circ} \mathrm{C}$ for 5 minutes. In the next step, the sample was moved at high vacuum using a shuttle (VCT100, Leica Microsystems) into the SEM (Magellan, FEI) equipped with a cold stage and the fractured structure was observed with $1 \mathrm{keV}$ electron beam at $-120^{\circ} \mathrm{C}$ and beam current $6.3 \mathrm{pA}$ without any metal coating.

Our results are quite encouraging, however, further systematic studies are required in order to fully monitor cell response mechanisms on applied stress. Such studies are currently under way in our laboratories, exploiting combination of SEM and Raman spectroscopy approaches. 


\section{References:}

[1] O. Samek et al, Sensors 10 (2010), p. 8635-8651.

[2] I. Marova et al, J of Environmental Management 95 (2012), p. S338.

[3] This work received support from the Ministry of Health, Ministry of Education, Youth and Sports of the Czech Republic (LO1212) together with the European Commission and the Czech Science Foundation (ALISI No. CZ.1.05/2.1.00/01.0017) and the Grant Agency of the Czech Republic (GA1420012S and GA15-20645S). A.H. and I.M. were supported by the project "Materials Research Centre at FCH BUT- Sustainability and Development" - no. LO1211 of the Ministry of Education, Youth and Sports of the Czech Republic. KH acknowledges the support of FEI/CSMS scholarship.
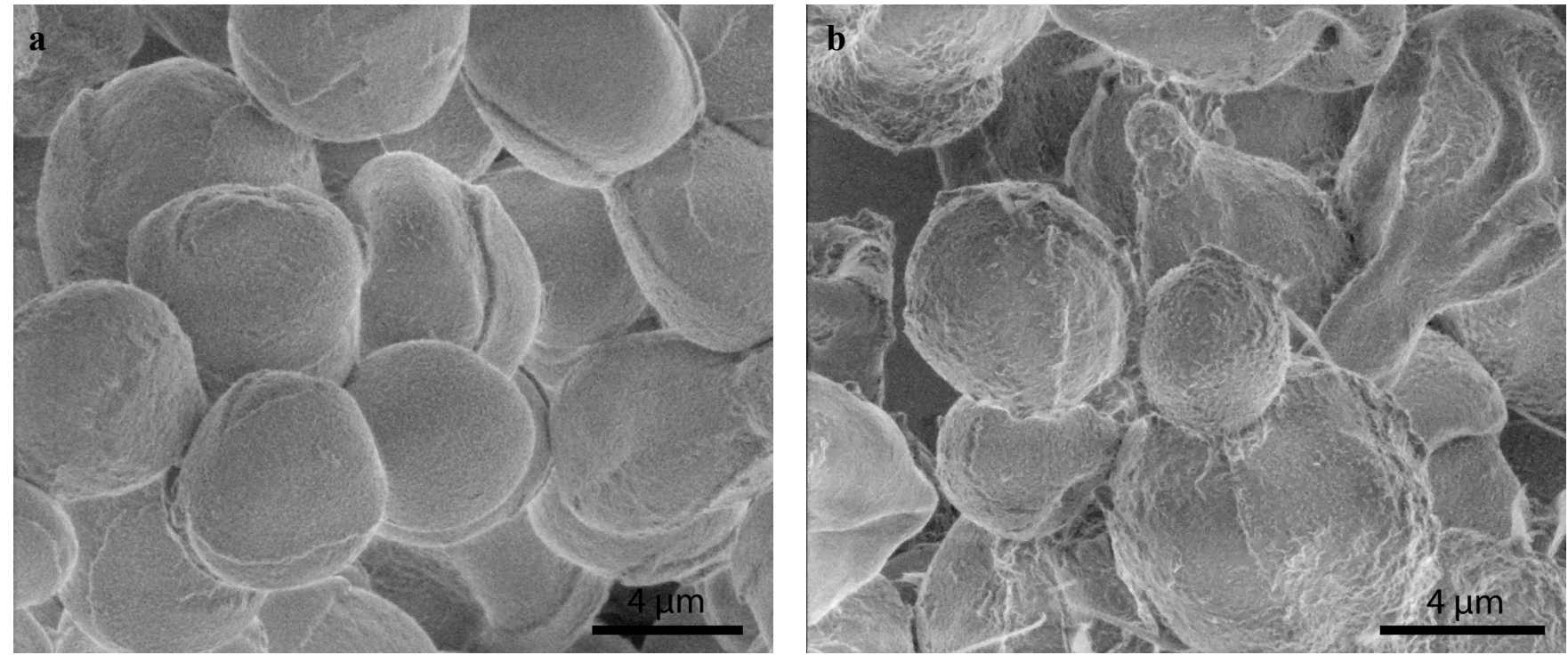

Figure 1. a) SEM image of Cystofilobasidium infirmominiatum (medium 1); b) SEM image of Cystofilobasidium infirmominiatum (medium 2), here different surface morphology is clearly visibly due to the applied stress.
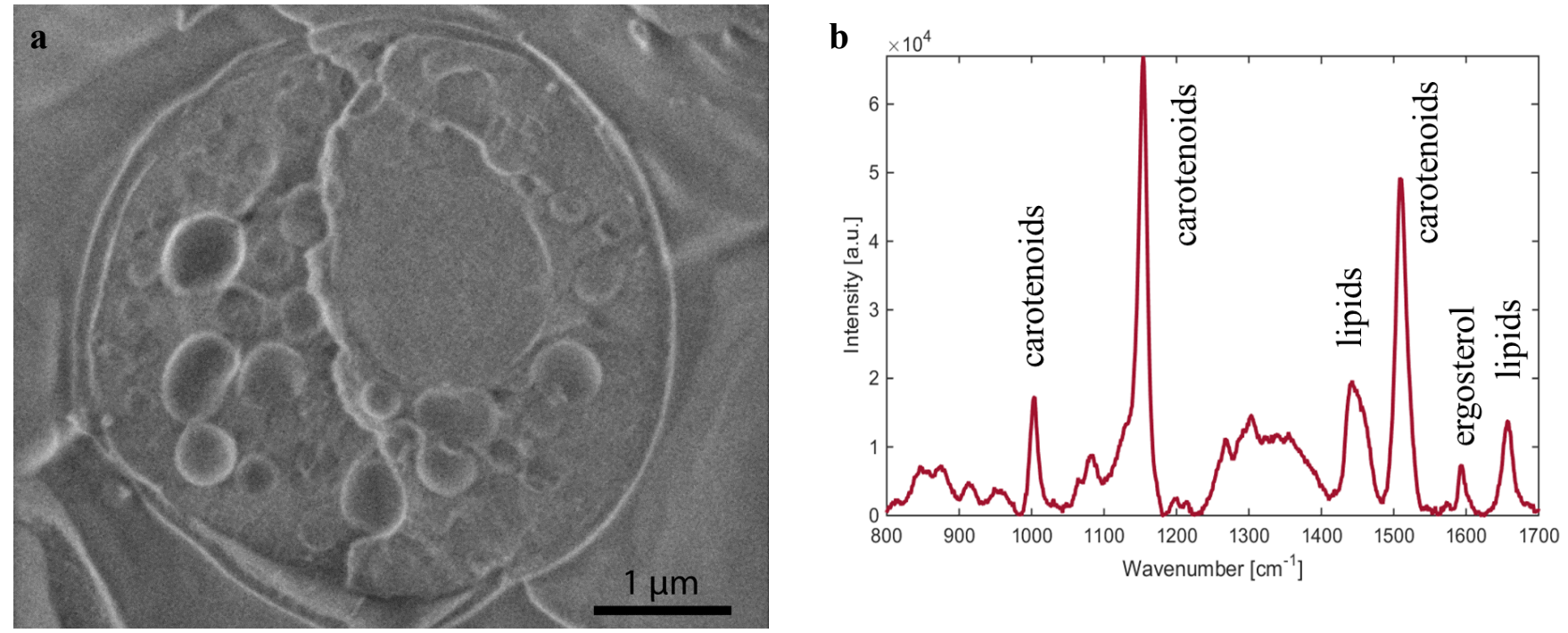

Figure 2. Sporobolomyces shibatanus (cultivated in lipid enhancing medium 2), a) Cryo-SEM image of one cell clearly showing lipid bodies, b) presence of applied stress on the same organism led to important overproduction of beta-carotene, ergosterol and lipids which can be confirmed by Raman spectroscopy. 